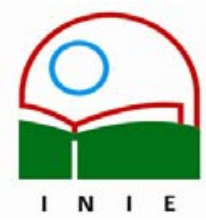

Actualidades Investigativas en Educación

Revista Electrónica publicada por el

Instituto de Investigación en Educación

Universidad de Costa Rica

ISSN 1409-4703

http://revista.inie.ucr.ac.cr

COSTA RICA

\title{
EVOLUCIÓN DE LA EDUCACIÓN SUPERIOR COSTARRICENSE: UN ANÁLISIS DE SU MODELO DE FINANCIACIÓN
}

THE RECENT EVOLUTION OF COSTA RICAN HIGHER EDUCATION: AN ANALYSIS OF

THE FUNDING MODEL

Volumen 7, Número 2

Mayo-Agosto 2007

pp. 1-23

Este número se publicó el 30 de agosto 2007

Susana Fernández Alfaro

Sara Fernández López

La revista está indexada en los directorios:

LATINDEX $\underline{\text { REDALYC}, ~ I R E S I E, ~ C L A S E, ~ D I A L N E T, ~ D O A J, ~ E-R E V I S T @ S, ~}$

La revista está incluida en los sitios:

REDIE, RINACE, OEI, MAESTROTECA, 


\title{
EVOLUCIÓN DE LA EDUCACIÓN SUPERIOR COSTARRICENSE: UN ANÁLISIS DE SU MODELO DE FINANCIACIÓN THE RECENT EVOLUTION OF COSTA RICAN HIGHER EDUCATION: AN ANALYSIS OF THE FUNDING MODEL
}

\author{
Susana Fernández Alfaro ${ }^{1}$ \\ Sara Fernández López ${ }^{2}$
}

\begin{abstract}
Resumen. Los Sistemas de Educación Superior (SES) de América Latina han atravesado por diversas etapas desde la expansión de la matrícula en los setenta, hasta las sucesivas crisis económicas y el deterioro de la calidad en los noventa, siendo el rasgo más característico la escasez de recursos de las universidades públicas. Para reducir estos problemas, los gobiernos adoptaron medidas orientadas fundamentalmente a la diversificación de las instituciones de la educación superior (IES), promoviendo la creación de instituciones privadas, la transformación de los mecanismos de financiación, y la implantación de los sistemas de evaluación y acreditación de la calidad.

El objetivo de este trabajo ha sido analizar la evolución de la educación superior de Costa Rica con el fin de comprobar en qué medida se han implantado estos procesos. Los resultados muestran que Costa Rica, al igual que el resto de países de la región, ha propiciado la aparición de instituciones privadas y recientemente está introduciendo cambios en el modelo de financiación de las universidades públicas que buscan reconducir la financiación dando mayor peso a los criterios próximos al mercado.
\end{abstract}

Palabras Clave: EDUCACIÓN SUPERIOR/ COSTA RICA/MODELOS DE FINANCIACIÓN/ PRIVATIZACIÓN/

\begin{abstract}
The Higher Education Systems (HES) of Latin America have gone through several stages from the growth of the enrolment in the 70's to the consecutive economic crises and the quality's deterioration in the 90's. As a consequence, the shortage of funds in public universities has become one of their most typical features. In order to reduce these problems the governments took several measures: the development of private higher education institutions (HEI), the change of funding models and the establishment of the quality's evaluation systems.

The objective of this work is to analyze the recent evolution of the Costa Rican Higher Education in order to know if these measures have been implemented. The results show that Costa Rican government, just like the rest of the Latin American governments, has created a favorable atmosphere for the development of private institutions. In addition, funding models of public universities are being changed with the purpose of reallocating the funds and attaching more importance to the market criteria.
\end{abstract}

Keyword: HIGHER EDUCATION/ COSTA RICA/ FUNDING MODELS/ PRIVATIZATION/

\footnotetext{
${ }^{1}$ Máster de Banca y Finanzas de la Universidad de Santiago de Compostela, España. Licenciada en Finanzas, Banca y Bolsa, de la Universidad Veritas, Costa Rica. Actualmente estudiante de doctorado y becaria del Grupo de Investigación "Valoración Financiera Aplicada (G-1866), Departamento de Economía Financiera y Contabilidad de la Universidad de Santiago, España. Correo electrónico susyfera@yahoo.com

2 Doctora en Ciencias Económicas por la Universidad de Santiago de Compostela; Licenciada en Ciencias Económicas y Empresariales, Especialista en Gestión de Cooperativas y Pymes. Actualmente es Profesora Contratada, Doctora de Economía Financiera y Contabilidad en las Facultades de Ciencias Empresariales de Santiago y Lugo, España. Su investigación se centra en Educación Superior (modelos de financiación, la aplicación de las TIC, desarrollo de patentes y emprendimiento, etc.) y en Finanzas y Seguros. Correo electrónico efsfl@usc.es
}

Artículo recibido: 24 de mayo, 2007

Aprobado: 28 de agosto, 2007 


\section{Introducción}

En la segunda mitad del siglo XX, especialmente en la década de los setenta, los sistemas de educación superior (SES) de América Latina se caracterizan por una espectacular expansión, producto del acceso libre a la formación universitaria. A partir de la década de los ochenta, los recursos que se destinan a la educación superior resultan insuficientes, lo que incentiva a los gobiernos de los respectivos países a emprender acciones para corregir dicha situación. Las medidas que han adoptado se resumen, fundamentalmente, en el fomento de la oferta privada, la transformación de los mecanismos de financiación y la implantación de los sistemas de evaluación y acreditación para mejorar la calidad del sistema universitario.

El objetivo de este trabajo ha sido analizar la situación particular de la educación superior costarricense, con el fin de comprobar la implantación de dichas reformas, especialmente las que hacen referencia a los modelos de financiación, y si estas contribuyen a corregir algunos de sus problemas. Para ello, el trabajo se estructura en seis apartados. En el primero se analiza la evolución de la educación superior, aportando algunas cifras referidas al crecimiento de la demanda y de la oferta. Luego se desarrollan en un segundo apartado las etapas, ofreciendo una panorámica general de los principales cambios institucionales y financieros, que permiten contextualizar la actual situación en la que se encuentra su educación superior. En un tercer apartado se considera el esfuerzo realizado por el gobierno en su financiación. Posteriormente, se analiza el financiamiento de las universidades públicas, centrando la atención en los modelos utilizados para asignar los recursos públicos y en los mecanismos que incentivan la diversificación de fondos. De la misma forma se procede a estudiar en el quinto apartado la financiación tanto pública como privada de los estudiantes. Finalmente, se resumen las principales conclusiones, así como las recomendaciones más importantes del presente trabajo.

\section{Evolución de la demanda y oferta de educación superior}

A partir de la década de los setenta, la educación superior de Costa Rica experimenta su mayor expansión como resultado del crecimiento demográfico que se produjo en la década de los cincuenta. En el Cuadro 1 se muestra la evolución de la matrícula de la educación superior según sector y subsistema para el periodo 1985-2004. Puede apreciarse como la cobertura de la educación superior ha sido creciente, pasando de una matrícula que no supera los 65.000 estudiantes en 1985, a casi triplicar esa cifra en 2004. A partir de la década de los noventa este crecimiento se atribuye principalmente a la participación de la 
educación universitaria y fundamentalmente de las instituciones pertenecientes al sector privado.

Cuadro $\mathrm{N}^{\circ} 1$

Evolución de la matrícula de la educación superior costarricense (1985-2004)

\begin{tabular}{|c|c|c|c|c|c|c|c|c|}
\hline \multirow{2}{*}{ Tipo de IES } & \multicolumn{2}{|c|}{1985} & \multicolumn{2}{|c|}{1990} & \multicolumn{2}{|c|}{2000} & \multicolumn{2}{|c|}{2004} \\
\hline & $N^{O}$ & $\%$ & $N^{\circ}$ & $\%$ & $N^{0}$ & $\%$ & $N^{0}$ & $\%$ \\
\hline IES Universitarias: & 58.393 & 92 & 67.132 & 90 & 133.755 & 94 & 166.417 & 95 \\
\hline Universidades Públicas & 50.033 & 78 & 54.999 & 74 & 61.654 & 43 & 75.974 & 43 \\
\hline Universidades Privadas & 8.360 & 14 & 12.133 & 16 & 72.101 & 51 & 86.951 & 50 \\
\hline Sin identificar & 0 & 0 & 0 & 0 & 0 & 0 & 3.492 & 2 \\
\hline IES No Universitarias $^{(\mathrm{a})}$ : & 5.378 & 8 & 7.594 & 10 & 7.874 & 6 & 8.867 & 5 \\
\hline Institutos parauniversitarios públicos & 1.939 & 3 & 2.098 & 3 & 5.011 & 4 & 6.779 & 4 \\
\hline Institutos parauniversitarios privados & 3.439 & 5 & 5.496 & 7 & 2.863 & 2 & 2.088 & 1 \\
\hline Total & 63.771 & 100 & 74.726 & 100 & 141.629 & 100 & 175.284 & 100 \\
\hline
\end{tabular}

(a) Los datos corresponden a registros administrativos del primer semestre o cuatrimestre

Fuente: Elaboración propia a partir de CONARE (2005)

En el periodo analizado, el sistema universitario atiende más del $90 \%$ de la matrícula total. El prestigio tradicional de las universidades podría explicar la preferencia de los estudiantes por cursar estudios superiores en estas instituciones, en lugar de hacerlo en las parauniversitarias, a pesar de la mayor orientación de estas hacia el mercado laboral. Las universidades de carácter público absorben en 1990 el 74\% de la matrícula en educación superior, porcentaje que se reduce a un $43 \%$ en los periodos siguientes, adquiriendo las universidades privadas un mayor peso, ya que pasan de concentrar el 13\% de la matrícula total registrada en 1990, a superar el 50\% en 2004.

El crecimiento de la matrícula ha supuesto la proliferación y diversificación de las instituciones de educación superior (IES), ya que hasta 1950 Costa Rica contaba con un total de dos instituciones (una universidad pública y una parauniversitaria ${ }^{2}$ ) (Ruiz, 2001b). Sin embargo, en 2003 este número es superado considerablemente al registrarse un total de 95 instituciones, gracias a la aparición de las privadas, tanto universitarias como no universitarias, en los noventa.

El Cuadro 2 muestra una tendencia creciente de las instituciones privadas, representando en 2003 el 88\% de las IES. Por el contrario, el comportamiento de las

${ }^{2}$ Su principal objetivo era ofrecer titulaciones cortas de dos o tres años de duración para la obtención del título de Diplomado y de Profesorado (Guadalupe y Louzano, 2002). 
instituciones públicas, muestra que las universidades han mantenido su número desde la década de los setenta, mientras que las parauniversitarias han experimentado un crecimiento moderado al pasar de 4 en 1985, a 7 en 2003.

\section{Cuadro $\mathrm{N}^{\circ} 2$}

Evolución de las IES costarricenses

\begin{tabular}{|c|c|c|c|c|c|c|c|c|c|c|}
\hline \multirow{2}{*}{ Tipo de IES } & \multicolumn{2}{|c|}{1985} & \multicolumn{2}{|c|}{1990} & \multicolumn{2}{|c|}{1995} & \multicolumn{2}{|c|}{2000} & \multicolumn{2}{|c|}{2003} \\
\hline & $N^{0}$ & $\%$ & $N^{O}$ & $\%$ & $N^{0}$ & $\%$ & $N^{0}$ & $\%$ & $N^{0}$ & $\%$ \\
\hline IES Universitarias & 6 & 33 & 12 & 35 & 28 & 42 & 53 & 55 & 54 & 57 \\
\hline Universidades públicas & 4 & 22 & 4 & 12 & 4 & 6 & 4 & 4 & 4 & 4 \\
\hline Universidades privadas & 2 & 11 & 8 & 23 & 24 & 36 & 49 & 51 & 50 & 53 \\
\hline IES No Universitarias & 12 & 67 & 22 & 65 & 38 & 58 & 44 & 45 & 41 & 43 \\
\hline Parauniversitarias públicas ${ }^{(a)}$ & 4 & 22 & 4 & 12 & 5 & 8 & 7 & 7 & 7 & 7 \\
\hline Parauniversitarias privadas & 8 & 45 & 18 & 53 & 33 & 50 & 37 & 38 & 34 & 36 \\
\hline Total & 18 & 100 & 34 & 100 & 66 & 100 & 97 & 100 & 95 & 100 \\
\hline
\end{tabular}

(a) Los Colegios Universitarios son la modalidad más generalizada en el país

Fuente: Elaboración propia a partir de MIDEPLAN

En general, las IES se concentran en el Valle Central (San José). No obstante, las universidades públicas, a diferencia de las privadas, han ampliado su cobertura a través de sedes (alrededor de 50) instaladas en diversas regiones del país.

\section{Evolución de la educación superior costarricense: 1970-actualidad}

La historia de la educación superior en Costa Rica es relativamente reciente, con aproximadamente 65 años de antigüedad ${ }^{3}$. La Universidad de Costa Rica (UCR), creada en 1940, asumiría en solitario la responsabilidad de la formación universitaria (Ruiz, 2001b), hasta la aparición en la década de los setenta de tres universidades públicas que vendrían a solventar la necesidad de plazas para responder al crecimiento de la demanda.

La evolución reciente de la educación superior costarricense se puede dividir en tres etapas: 1) de 1970 a 1980, cuya característica es la expansión y consolidación de las universidades públicas; 2) de 1980 a 1990, cuando la crisis económica por la que atraviesa el país propicia la creación y proliferación de las IES privadas, y 3) de 1990 hasta la

${ }^{3}$ La Casa de Enseñanza de Santo Tomás, primera IES creada en el siglo XIX, tuvo una corta duración ya que desaparece en 1888, dando lugar a las Escuelas Superiores Profesionales (Brenes, 2005). 
actualidad, periodo que contempla las principales reformas orientadas a la mejora de la calidad del sistema universitario, tal y como mostramos en la Figura 1.

Figura $\mathrm{N}^{\circ} 1$

Etapas en el proceso de cambio de la educación superior costarricense

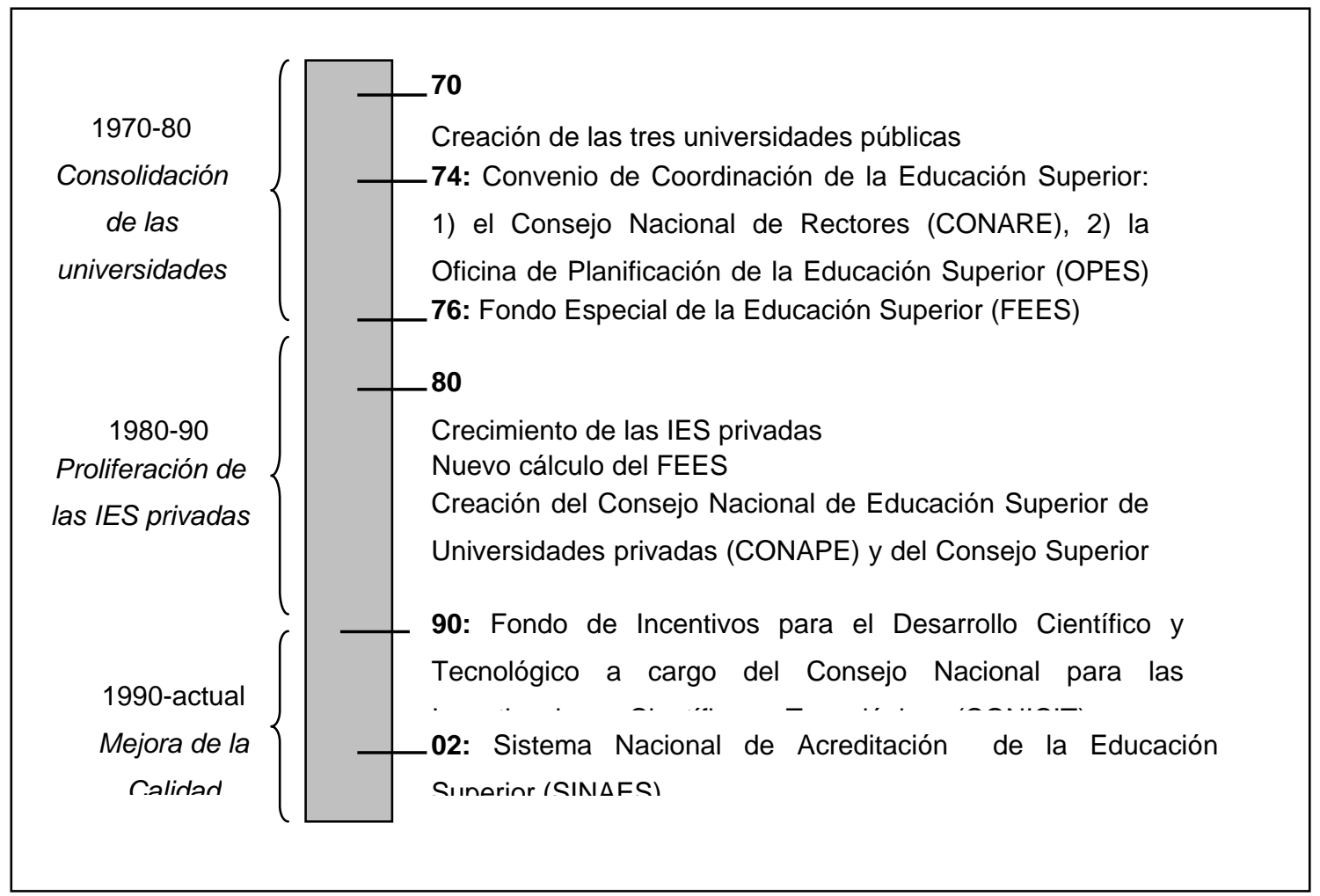

Seguidamente se realiza una breve reseña de las etapas de la educación superior en

Costa Rica, resaltando las reformas más importantes.

\subsection{0-1980: Consolidación de las universidades públicas}

En la década de los cincuenta del siglo XX, el país experimentó un fuerte crecimiento demográfico que generó, a su vez, una presión por espacios universitarios a finales de los sesenta y, principalmente, en los setenta. Esta situación contribuyó al crecimiento de la demanda de educación superior a lo cual la UCR, como única universidad existente, no estaba en condiciones de responder satisfactoriamente, lo que provocó la aparición de nuevas universidades públicas y sedes regionales ${ }^{4}$ con el fin de democratizar la educación superior. Así, en la década de los setenta se unen a la UCR tres universidades públicas: el

${ }^{4}$ En 1968 la UCR crea en San Ramón su primera sede regional (CONARE, 2005). 
Instituto Tecnológico de Costa Rica, la Universidad Nacional y la Universidad Estatal a Distancia $^{5}$, y una universidad privada denominada Universidad Autónoma de Centroamérica, esta última creada a finales de la década.

La característica más sobresaliente de esta etapa es, por tanto, la consolidación de las universidades públicas. Estas reciben recursos estatales, siendo el Ministerio de Educación Pública el responsable de su distribución. No obstante, dichos recursos llegaron a resultar insuficientes para enfrentar las múltiples necesidades institucionales, ya que no existía una política por parte del Estado que asegurase a las universidades una financiación estable y suficiente.

A raíz de esta preocupante situación, las universidades se unen para negociar con las autoridades gubernamentales lo referente a su financiación. Es así como surge en 1974, por iniciativa de las propias instituciones, el primer Convenio de Coordinación de la Educación Superior, dando origen a nuevos organismos de coordinación y planificación de las universidades públicas, tales como el Consejo Nacional de Rectores (CONARE), integrado por los rectores de las universidades, la Oficina de Planificación de la Educación Superior (OPES), como órgano técnico del CONARE, y la Comisión de Enlace, constituida en ese momento por los rectores y los Ministros de Educación, Hacienda, Planificación y de Ciencia y Tecnología (Araya, 2003).

Dos años después, en 1976, se crea el Fondo Especial para la Educación Superior (FEES) mediante la Ley de Reforma Tributaria $N^{0} 5909 / 1976{ }^{6}$, que surge como un mecanismo financiero para solventar las necesidades de las universidades estatales, estableciendo para ello su composición y los procedimientos para su distribución.

\subsection{0-1990: Proliferación de las IES privadas}

La crisis económica de principios de los ochenta generó graves problemas en la financiación de la educación pública; el presupuesto de la educación disminuyó, pasando del 5,6\% del PIB en 1980 al 4,04\% en 1989. La educación preuniversitaria fue la principal

\footnotetext{
${ }^{5}$ La UNED pretendía, con la utilización de metodologías menos tradicionales y siguiendo experiencias de países europeos y latinoamericanos (Gran Bretaña, España, Méjico y Venezuela), expandir y dirigir la educación a nuevos sectores de la población (sector rural) y a los trabajadores sometidos a horarios rígidos. De hecho, es la universidad que más sedes tiene distribuidas en el país (Araya, 2003).

${ }^{6}$ El artículo 7 establece que el FEES estará compuesto por el impuesto de sociedades, el impuesto sobre el traspaso de bienes inmuebles, y entre el $25 \%$ y $30 \%$ del impuesto sobre la renta, los cuales serán distribuidos conforme a las normas y principios establecidos en el Convenio de Coordinación (CONARE, 2003).
} 
víctima, ya que el porcentaje de PIB destinado a este nivel educativo se redujo en 1,7 puntos porcentuales entre 1980 y 1989, con una tendencia decreciente a lo largo de la década. Por el contrario, el porcentaje de PIB destinado a gasto público en educación superior muestra una tendencia creciente hasta 1986 (Ruiz, 2001a). No obstante, la crisis en este nivel educativo se deja sentir a partir de 1987, cuando surge la necesidad de reorientar los recursos hacia la educación primaria y secundaria, provocando una disminución del monto del FEES en términos reales. Con el fin de corregir esta situación se propone, a finales de los ochenta, un nuevo cálculo del FEES, tal y como veremos al analizar la financiación de la educación superior.

Las dificultades que experimentan las universidades públicas, así como su escasa capacidad para responder a las demandas de la sociedad y su rigidez en los procesos académicos y administrativos, contribuyeron a la proliferación de universidades privadas, situación que adquiere una enorme dimensión en la década de los noventa (CONARE, 2005). Aunque la expansión del sector privado permitió atender a los estudiantes excluidos del sector público, tuvo efectos perjudiciales para las universidades públicas, ya que el Estado se inclina a favor de disminuir sus recursos y promover el desarrollo del sector privado (Ruiz, 2001c).

En general, los cambios en la década de los ochenta tuvieron su particular efecto sobre la estructura de la educación superior que se vuelve más compleja, integrando las universidades privadas y las instituciones de carácter no universitario o parauniversitario creadas en la década anterior para incrementar las posibilidades de ingreso en la educación superior. Por ello es necesario la creación de organismos encargados de su regulación, como el Consejo Nacional de Educación Superior de Universidades Privadas (CONESUP) y el Consejo Superior de Educación, que estaría a cargo de las IES parauniversitarias tanto públicas como privadas (Mora, 2006). Por otro lado, las reformas también afectaron la financiación, al establecerse cambios en el FEES para mejorar la situación económica de las universidades públicas.

Pese a las debilidades sociopolíticas que llevaron al país a la crisis económica en los ochenta y, en consecuencia, redujeron las posibilidades y oportunidades educativas, el comportamiento del gobierno muestra un interés por mejorar y elevar el gasto público en el 
sector educativo, planteando como objetivo el aumento del porcentaje de PIB destinado a educación, objetivo que hasta la fecha no ha logrado alcanzar ${ }^{7}$.

\subsection{0-actual: Mejora de la calidad}

En esta etapa son importantes los esfuerzos realizados para llevar a cabo actividades de investigación en el país. El Ministerio de Ciencia y Tecnología, por medio del Consejo Nacional para Investigaciones Científicas y Tecnológicas, creado en 1972, es el encargado de llevar a la práctica la Ley de Promoción al Desarrollo Científico y Tecnológico № 7169 , aprobada en 1990. La ley establece la creación de un Fondo de Incentivos para el Desarrollo Científico y Tecnológico con el objeto de incentivar a los docentes que realizan proyectos y actividades de investigación en ciencia y tecnología, pero se debe competir por dichos fondos.

Por su parte, el crecimiento del sistema universitario privado no estuvo acompañado de un marco de regulación y vigilancia de la calidad académica. Como consecuencia, surgen los instrumentos destinados a la evaluación y acreditación de la calidad de las universidades, así como de sus titulaciones y programas de estudio. De este modo, el Sistema Nacional de Acreditación de la Educación Superior (SINAES) fue aprobado mediante el Decreto Legislativo № 8256 en abril de 2002, hecho que coincide con un periodo de estabilidad en la expansión universitaria privada. Dicha ley declara las actividades del SINAES de "interés público" y señala, en su artículo 2, que la acreditación tiene como propósito identificar con carácter oficial las titulaciones y programas de las universidades públicas y privadas que cumplan los requisitos de calidad establecidos (Mora, 2005).

EI SINAES cuenta con un Consejo Nacional de Acreditación de la Educación Superior que realiza la certificación de la calidad de las titulaciones de las universidades. Tres etapas caracterizan el proceso de acreditación (Alvarado, 2005): a) autoevaluación institucional, b) evaluación externa y c) evaluación final. La acreditación que se desarrolla a través de este instrumento es voluntaria, proceso al que se encuentran adscritas las cuatro universidades públicas y siete de las universidades privadas. Estas universidades agrupan a un porcentaje mayoritario de la matrícula de educación superior, lo que hace suponer la relevancia que se

\footnotetext{
${ }^{7}$ El artículo 78 de la Constitución Política establece que "el gasto público dedicado a la educación estatal no debe ser inferior al 6\% del PIB" (Conejo, 2004).
}

Volumen 7, Número 2, Año 2007, ISSN 1409-4703 
le otorga a las titulaciones acreditadas. Hasta mayo de 2005, el SINAES había acreditado 24 titulaciones universitarias y 17 se encontraban en proceso de acreditación (Mora, 2005).

\section{El esfuerzo financiero}

La educación pública tuvo un crecimiento sostenido entre los años 1949 y 1980, situación que coincidió con la abolición del ejército en 1948, lo cual podría explicar tal crecimiento $^{8}$ (Ruiz, 2001a). Así, el presupuesto del Ministerio de Educación Pública alcanzó el 5,73\% del PIB en 1979. Esta tendencia se ve paralizada por la crisis de la década de los ochenta, ya que en este periodo el gasto público en educación sufrió una caída extraordinaria que osciló entre el 4,75\% y el 4,04\% del PIB (cifras que corresponden a los años 1981 y 1989, respectivamente). En la década siguiente, el porcentaje de PIB destinado al gasto público en educación muestra un lento pero constante crecimiento, pasando del 3,8\% en 1990 al 4,9\% en 2003, siendo significativamente elevado el resultado de 2002 (5,5\%). En este mismo periodo, el porcentaje de PIB destinado al gasto público en educación superior se redujo, pasando de un 1\% en 1990 a un 0,9\% en 2003 (Cuadro 3).

\section{Cuadro $\mathrm{N}^{\circ} 3$}

Gasto público en educación y educación superior como porcentaje del PIB y del gasto público (1990-03)

\begin{tabular}{|c|c|c|c|c|c|c|c|}
\cline { 2 - 7 } & \multicolumn{4}{|c|}{ Gasto Público en Educación (\%) } & \multicolumn{2}{c|}{$\begin{array}{c}\text { G. pco. Educación } \\
\text { Superior/G. pco. } \\
\text { Sducación }\end{array}$} \\
\cline { 2 - 7 } & \multicolumn{3}{|c|}{ PIB } \\
\cline { 2 - 7 } & Básica & Superior & Total & Básica & Superior & Total & \multicolumn{2}{c|}{ Easto Público } \\
\hline 1990 & 2,8 & 1,0 & 3,8 & 20,4 & 7,6 & 28 & 27 \\
\hline 1995 & 2,9 & 0,9 & 3,8 & 18 & 6,0 & 24 & 25 \\
\hline 2000 & 3,9 & 0,8 & 4,7 & 25,4 & 5,6 & 31 & 18 \\
\hline 2003 & 4,0 & 0,9 & 4,9 & 24,6 & 5,4 & 30 & 18 \\
\hline
\end{tabular}

(a) Estimado a partir de los datos obtenidos

Fuente: Elaboración propia a partir de Conejo (2004)

Por su parte, la prioridad que el gobierno concede a la educación dentro de los presupuestos públicos produce un moderado crecimiento, pasando de representar un $28 \%$ en 1990 a un 30\% en 2003. De dicho presupuesto educativo se ha asignado más de un $70 \%$ a financiar el nivel básico, con una tendencia creciente en el periodo analizado. Por el

${ }^{8}$ Según estadísticas de la Comisión Económica para América Latina (CEPAL), Costa Rica asignó en la década de los setenta, en promedio, un 5,3\% del PIB a educación, superando al resto de países de la región, exceptuando a Cuba para el cual no se tiene información (www.cepal.org/estadísticas). 
contrario, la participación de la educación superior en el presupuesto educativo ha descendido, pasando del $27 \%$ a principios de los noventa al 18\% en 2003, lo que indica la prioridad del gobierno por financiar los niveles inferiores (Conejo, 2004).

Este comportamiento del gasto público responde a las recomendaciones lanzadas durante la década de los noventa desde diferentes organismos internacionales, entre los que destacan el Banco Mundial y el Fondo Monetario Internacional, quienes para los países en vías de desarrollo una mayor inversión en los niveles inferiores en detrimento de la educación superior. Así, el Banco Mundial (1995) afirma que la educación superior es menos rentable desde el punto de vista social que los niveles inferiores y, en consecuencia, sostiene que la educación superior no debería tener la consideración de prioritaria en los incrementos del gasto público en muchos países en desarrollo donde la primaria y la secundaria no han alcanzado niveles adecuados de acceso, equidad y calidad.

Como consecuencia de esta orientación, las universidades no pueden depender exclusivamente del presupuesto estatal si esperan cumplir con las tareas necesarias para alcanzar sus objetivos. Por ello, la necesidad de generar recursos adicionales es cada vez más patente en el medio universitario.

\section{La financiación de las universidades públicas}

Las universidades públicas han sido financiadas desde 1949 bajo el amparo de la Constitución Política de la República. Inicialmente, el artículo 85 incorpora la independencia económica de la UCR con una asignación no menor al 10\% del presupuesto anual del Ministerio de Educación Pública, al mismo tiempo que define su autonomía universitaria ${ }^{9}$ y su libertad de cátedra (Ruiz, 2001a). Posteriormente, en 1977 la ley se modifica (mediante Ley $N^{\circ}$ 6052) a fin de cubrir también a las nuevas universidades públicas, extendiendo su garantía presupuestaria y definiendo los procedimientos por seguir. Así, el artículo 85 de la Constitución Política establece que:

El Estado dotará de patrimonio propio a la Universidad de Costa Rica, al Instituto Tecnológico de Costa Rica, a la Universidad Nacional y a la Universidad Estatal a Distancia y les creará rentas propias, independientemente de las originadas en estas instituciones. Además mantendrá, con las rentas actuales y con otras que sean

\footnotetext{
${ }^{9}$ La autonomía se consignó por primera vez en el Estatuto Orgánico de la UCR en 1940, la cual establece a este respecto que "las universidades del Estado están dotadas de independencia para el desempeño de sus funciones y de plena capacidad jurídica para adquirir derechos y contraer obligaciones, así como para darse su organización y gobierno propios" (Mora, 2005; p. 50).
} 
necesarias, un fondo especial para la financiación de la educación superior pública

(Calderón, 2005, p. 52).

Hasta mediados de la década de los setenta, los aportes estatales se definían mediante procesos de negociación entre la administración y las universidades públicas, que se basaban en el incremento anual de presupuestos anteriores. Posteriormente, la Comisión de Coordinación de la Educación Superior establece que el monto del FEES será asignado en función de inputs como el número de estudiantes y el costo de los programas académicos ofertados por cada institución.

Además de los aportes públicos, las universidades pueden recibir fondos del sector privado procedentes del cobro de matrículas, donaciones, convenios y venta de bienes y servicios (Conejo, 2004).

El Gráfico 1 muestra la composición de los recursos totales de las universidades públicas para el año 2003. Los fondos públicos representan el 85\%, lo que indica que el 15\% restante proviene de los fondos privados. Dentro de estos, el importe por concepto de matrículas representa el 33\% y suelen utilizarse para otorgar becas a los estudiantes.

\section{Gráfico $N^{\circ} 1$}

\section{Origen de los fondos de las universidades públicas (distribución porcentual) (2003)}

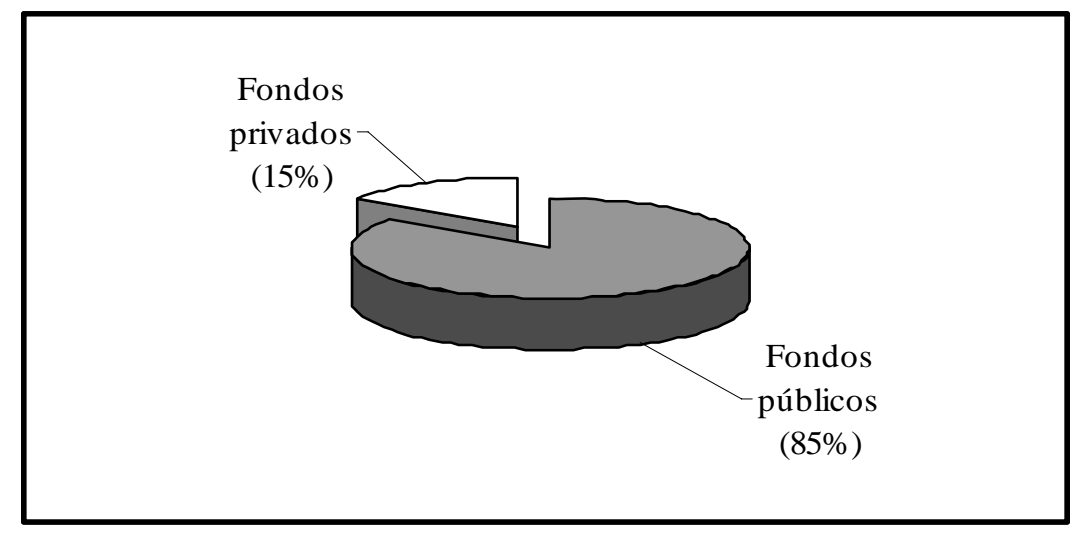

Fuente: Elaboración propia a partir de Conejo (2004)

En el caso de las universidades e instituciones privadas, no reciben apoyo financiero del gobierno y, por lo tanto, deben generar sus propios recursos por la vía del cobro de matrículas y la venta de servicios educativos. 


\subsection{Fondos públicos}

En 1987 y 1988 el presupuesto del FEES resulta insuficiente para hacer frente al crecimiento de la demanda y al desempeño adecuado de las actividades institucionales, ya que los montos girados fueron decrecientes en términos reales, lo que llevó a las universidades a negociar recursos adicionales con el Estado. Una de las estrategias empleadas por el gobierno para aliviar la disminución del presupuesto público fue asignar recursos a las universidades que incrementasen sus recursos propios. Ante esta situación el CONARE en 1988 propuso al gobierno la creación del I Convenio de Financiación de la Educación Superior que regiría para el quinquenio 1989-93 (Conejo, 2004), siendo la Comisión de Enlace la responsable de aprobar cada cinco años los convenios siguientes, con la inclusión de algunas modificaciones en función de las necesidades del momento (ver Cuadro 4).

\section{Cuadro $\mathrm{N}^{\circ} 4$}

Reformas financieras al Convenio de Financiación de la Educación Superior

\begin{tabular}{|c|c|c|c|c|}
\hline ELEMENTOS & $\begin{array}{c}\text { I CONVENIO } \\
\text { (1989-1993) }\end{array}$ & $\begin{array}{c}\text { II CONVENIO } \\
\text { (1994-1998) }\end{array}$ & $\begin{array}{l}\text { III CONVENIO } \\
\text { (1999-2003) }\end{array}$ & $\begin{array}{c}\text { IV CONVENIO } \\
(2005-2009)\end{array}$ \\
\hline $\begin{array}{c}\text { REAJUSTE } \\
\text { AUTOMÁTICO } \\
\text { DEL FEES }\end{array}$ & $\begin{array}{l}\text { El monto anual } \\
\text { del FEES re } \\
\text { calcula como el } \\
\text { del año anterior } \\
\text { más r un } \\
\text { incremento con } \\
\text { base en la } \\
\text { variación del IPC } \\
\text { de los años (t) y } \\
\text { (t-1) }\end{array}$ & $\begin{array}{l}\text { El reajuste del } \\
\text { FEES es similar } \\
\text { al aplicado en el } \\
\text { quinquenio } \\
\text { anterior, la única } \\
\text { diferencia es que } \\
\text { considera } \\
\text { únicamente la } \\
\text { variación del IPC } \\
\text { para el año (t) }\end{array}$ & $\begin{array}{l}\text { El reajuste del } \\
\text { FEES se efectúa } \\
\text { considerando el } \\
\text { IPC del año (t), la } \\
\text { tasa de crecimiento } \\
\text { de la población } \\
\text { total del país y la } \\
\text { participación en el } \\
\text { crecimiento } \\
\text { económico (PIB } \\
\text { real/habitantes) }\end{array}$ & $\begin{array}{l}\text { El monto anual del } \\
\text { FEES se calcula } \\
\text { como un porcentaje } \\
\text { creciente del PIB } \\
\text { según proyecciones } \\
\text { del Banco Central }\end{array}$ \\
\hline $\begin{array}{c}\text { DISTRIBUCIÓ } \\
\text { N DEL FEES }\end{array}$ & \multicolumn{2}{|c|}{$\begin{array}{l}\text { Se determina un porcentaje del monto } \\
\text { del FEES que será asignado en } \\
\text { función de los recursos privados que } \\
\text { generan las universidades }\end{array}$} & \multicolumn{2}{|c|}{$\begin{array}{l}\text { No indican el monto del FEES que se } \\
\text { destina a incentivar la diversificación de } \\
\text { fondos }\end{array}$} \\
\hline $\begin{array}{l}\text { RECURSOS } \\
\text { ADICIONALES }\end{array}$ & $\begin{array}{l}\text { Recursos } \\
\text { aprobados por } \\
\text { la Comisión de } \\
\text { Enlace } \\
\text { función de los } \\
\text { requerimientos } \\
\text { de las } \\
\text { universidades, } \\
\text { por ejemplo la } \\
\text { revisión de los } \\
\text { salarios, la Ley } \\
\text { de Protección al } \\
\text { Trabajador y el } \\
\text { salario escolar } \\
\end{array}$ & $\begin{array}{l}\text { El gobierno } \\
\text { destina al Fondo } \\
\text { de Renovación de } \\
\text { Equipo Científico } \\
\text { y Tecnológico el } \\
\text { equivalente al } 2 \% \\
\text { anual del monto } \\
\text { del FEES. Las } \\
\text { universidades } \\
\text { deben aportar } \\
\text { sumas iguales a } \\
\text { las recibidas para } \\
\text { el desarrollo de } \\
\text { dicho programa }\end{array}$ & \multicolumn{2}{|c|}{$\begin{array}{l}\text { El gobierno y las universidades mantienen } \\
\text { su compromiso con los recursos } \\
\text { establecidos en el primer convenio y con } \\
\text { el Fondo de Renovación de Equipo } \\
\text { Científico y Tecnológico, en las mismas } \\
\text { condiciones estipuladas en los } \\
\text { quinquenios anteriores }\end{array}$} \\
\hline
\end{tabular}




\begin{tabular}{|c||l|l|}
\hline $\begin{array}{c}\text { RECURSOS } \\
\text { PROPIOS }\end{array}$ & $\begin{array}{l}\text { Las universidades se comprometen } \\
\text { a generar un porcentaje de recursos } \\
\text { privados }\end{array}$ & $\begin{array}{l}\text { Se mantiene el compromiso en la } \\
\text { captación de dichos recursos aunque no } \\
\text { se establece un porcentaje determinado }\end{array}$ \\
\hline
\end{tabular}

Fuente: Elaboración propia a partir de Conejo (2004)

Los elementos financieros que se incorporan en el I Convenio de Financiación son los siguientes:

a) El reajuste automático del FEES ${ }^{10}$. Los cambios en el convenio han permitido mantener el monto del FEES de acuerdo con el poder adquisitivo de la moneda, condición que establece el artículo 5 de la Ley 5909 de creación del FEES. Se considera para el reajuste del monto del FEES el índice de inflación y el PIB.

b) La distribución del FEES. Asigna recursos a las universidades en función, por un lado, del número de estudiantes y los costos de los programas académicos, y por otro, del volumen de recursos privados generados por estas, estableciendo el CONARE la parte del FEES que sería destinado a este fin. Así, en 1989 este porcentaje es del 2\% y se incrementa cada año en otro 2\% hasta alcanzar en 1993 un 10\% de los recursos del FEES, manteniéndose durante el Convenio II en un 10\% para cada año. En los dos últimos convenios no se indica el monto del FEES que irá destinado a incentivar la captación de recursos privados.

c) La asignación de recursos adicionales. Se asignan recursos adicionales para proyectos específicos a cargo de la Comisión de Enlace. Estos fondos adicionales se determinan de acuerdo con el requerimiento de cada institución ${ }^{11}$, por ejemplo la revisión del salario profesional, la Ley de protección al trabajador y el salario escolar.

d) La generación de recursos propios. Las universidades públicas se comprometen a seguir aumentando progresivamente la cuantía de sus recursos propios. En los dos primeros convenios se establecen los porcentajes que deben captar anualmente las universidades, los cuales van creciendo desde el 8,8\% del monto del FEES en 1989 hasta el 14\% en 1998 (Conejo, 2004). Dicho compromiso se mantiene en los últimos convenios, aunque en ellos no se indican los porcentajes que han de recaudar.

Las modificaciones en dichos convenios han sido mínimas, sin embargo, debemos destacar el último, correspondiente al quinquenio 2005-2009 ya que abandona el mecanismo

\footnotetext{
${ }^{10}$ Se utiliza como indicador el Índice de Precios al Consumidor (IPC) de los ingresos medios y bajos del Área Metropolitana de San José.

${ }^{11}$ Estos recursos han beneficiado en más de un 55\% a la UCR, seguida del Instituto Tecnológico de Costa Rica con alrededor de un 23\% (Cervantes, 1999).
} 
de reajuste automático en función del índice de inflación y utiliza el PIB como variable para definir el monto del FEES. Así, según las proyecciones del Banco Central de Costa Rica, se define un porcentaje creciente del PIB que va desde 0,9\% en 2005, a 1,05\% en 2009. Posteriormente a la suscripción del IV Convenio $^{12}$ (julio 2004), el CONARE modifica en septiembre de ese año los criterios para la distribución de los fondos del FEES, que integraría dos partidas presupuestarias (CONARE, 2005; Barquero et al., 2006):

1) Un Fondo Institucional (FI) destinado a cubrir las necesidades básicas de las universidades. El FI toma como base el presupuesto histórico y lo incrementa en función de la población estudiantil y de los costes de los diversos programas (inputs). Para ello, cada universidad elabora su presupuesto y lo remite a la Contraloría General de la República quien es finalmente la encargada de aprobar su ejecución (Conejo, 2004).

2) Un Fondo del Sistema (FS), cuyos recursos están condicionados al cumplimiento de objetivos que han sido establecidos para el desarrollo de actividades o proyectos específicos que promueven la construcción y fortalecimiento de la educación superior pública. El FS se establece como un porcentaje de los recursos adicionales, los cuales se obtienen de la diferencia entre el monto del FEES calculado de acuerdo con el nuevo convenio y el monto que resultaría de la aplicación del mecanismo establecido en el convenio anterior. Así, para el quinquenio 2005-09, los fondos que se destinan al FS representan en 2005 el 25\% de los recursos adicionales y alcanzarán el 50\% en 2009 (CONARE, 2005).

Además de los fondos del FEES, el CONARE mantiene los aportes correspondientes a las obligaciones derivadas de acuerdos previos (salario escolar, Ley de protección al Trabajador y el Fondo de Renovación de Equipo Científico y Tecnológico), así como el compromiso por parte de las universidades públicas en la generación de recursos privados.

En el Cuadro 5 se muestra la estructura del FEES y su distribución entre las universidades públicas para los años 2005 a 2007. Así, la UCR recibe el 57\%, la Universidad Nacional el $23 \%$ y el Instituto Tecnológico de Costa Rica el 11\%, teniendo la Universidad Estatal a Distancia una asignación que no supera el 10\%. Además de las universidades, el CONARE recibe un 0,9\% del presupuesto del FEES (Conejo, 2004).

\footnotetext{
${ }^{12}$ El III Convenio, con vencimiento a noviembre 2003, se prorroga hasta que se firma el nuevo acuerdo (CONARE, 2006).
} 


\section{Cuadro $\mathrm{N}^{\circ} 5$}

Distribución del monto del FEES entre las universidades (en millones de colones)

\begin{tabular}{|l|c|c|c|c|c|c|}
\hline \multirow{2}{*}{ CONCEPTO $^{(a)}$} & \multicolumn{2}{c|}{2005} & \multicolumn{2}{c|}{2006} & \multicolumn{2}{c|}{2007} \\
\cline { 2 - 7 } FONDO INSTITUCIONAL & Monto & $\%$ & Monto & $\%$ & Monto & $\%$ \\
\hline Universidad de Costa Rica (UCR) & $\mathbf{8 6 . 0 0 9 , 4 1}$ & $\mathbf{9 9}$ & $\mathbf{9 8 . 5 1 9 , 3 3}$ & $\mathbf{9 8 , 8}$ & $\mathbf{1 2 2 . 5 3 5 , 1}$ & $\mathbf{9 5 , 8}$ \\
\hline Universidad Nacional (UNA) & $49.096,26$ & 57 & $56.219,72$ & 57 & $69.896,8$ & 57 \\
\hline $\begin{array}{l}\text { Instituto Tecnológico de Costa } \\
\text { (ITCR) }\end{array}$ & $19.876,68$ & 23 & $22.764,17$ & 23 & $28.302,2$ & 23 \\
\hline Universidad Estatal a Distancia (UNED) & $9.599,71$ & 11 & $10.992,96$ & 11 & $13.667,3$ & 11 \\
\hline CONARE & $6.373,54$ & 8 & $7.305,94$ & 8 & $9.083,3$ & 8 \\
\hline FONDO DEL SISTEMA & $1.063,22$ & 1 & 1236,54 & 1 & $1.585,5$ & 1 \\
\hline Total & $\mathbf{5 7 4 , 9 1}$ & $\mathbf{1}$ & $\mathbf{1 . 2 0 1 , 7 3}$ & $\mathbf{1 , 2}$ & $\mathbf{5 . 3 2 8 , 3}$ & $\mathbf{4 , 2}$ \\
\hline
\end{tabular}

(a) Puede incluir partidas acordadas en años anteriores

Fuente: Elaboración propia a partir de CONARE (2005)

En el Cuadro 5 se aprecia que en los tres periodos considerados, más del 95\% del presupuesto público a la educación superior se canaliza a través del FI, ya que el FS, aunque muestra un crecimiento significativo que prácticamente se multiplica por ocho, representa menos de un 5\% del total del FEES, lo que podría explicarse por su reciente implantación.

Adicionalmente, el CONARE conforma un Fondo Concursable sobre el Estado de la Educación para $2007^{13}$, por un monto de 50 millones de colones para promover la investigación sobre temas relevantes de la política educativa costarricense en las universidades públicas. El Fondo Concursable tiene un carácter retrospectivo, ya que realiza los desembolsos una vez alcanzados los objetivos fijados en el cronograma de los proyectos presentados por las universidades, siendo penalizadas en caso de incumplimiento.

Cervantes (1999) hace referencia a un estudio de Jofré, exrector del Instituto Tecnológico de Costa Rica, quien manifiesta que el Convenio de Financiación de la Educación Superior ha presentado enormes beneficios para el sistema universitario público del país. Por un lado, ha permitido eliminar las largas negociaciones entre rectores y Ministros, dotando a las universidades de autonomía financiera en la planificación de los recursos recibidos $y$, por otro lado, ha concentrado sus esfuerzos, con el fin de lograr recursos adicionales para el desarrollo universitario.

\footnotetext{
${ }^{13}$ Este fondo es administrado por el Programa Estado de la Nación.
} 


\subsection{Fondos privados}

La crisis de la década de los ochenta generó cambios sustanciales en la estructura financiera de las universidades públicas en respuesta a la carencia de recursos para enfrentar el crecimiento de la demanda. En este sentido, los dos primeros Convenios de Financiación para la Educación Superior establecen el compromiso de las universidades públicas en la generación de recursos propios (ver Cuadro 5). En el quinquenio 1989-93 equivalían, en promedio, a un 10\% del monto del FEES, mientras que en el quinquenio 199498 llegaron a alcanzar hasta un 14\% (CONARE, 2003).

En los dos últimos quinquenios, se mantiene el compromiso de las universidades en la captación de recursos propios, pero no se les exige cantidades determinadas. En 2003, los fondos privados, tal y como vimos en el Gráfico 1, representan un 15\% del presupuesto total de las universidades públicas, correspondiendo el 33\% al cobro de matrícula y el 67\% a la venta de bienes y servicios educativos y otros.

\section{La financiación de los estudiantes}

En Costa Rica, los estudiantes de la educación superior no disponen de ayudas financieras suficientes que les permitan sufragar los costos de su formación, situación que limita sus posibilidades de acceso. A continuación veremos las principales modalidades de ayuda en función de la fuente de financiación pública o privada.

\subsection{Fondos públicos}

Los recursos públicos se canalizan a los estudiantes mediante un sistema de becas del Ministerio de Educación Pública. Este programa, denominado Fondo Nacional de Becas (FONABE) (Ley No 7658), beneficia a estudiantes de los diversos niveles educativos, incluida la educación superior, al atorgar recursos para la realización de estudios dentro y fuera del país. Las becas son adjudicadas por una Junta Directiva que toma en consideración los méritos, el rendimiento académico y la situación socioeconómica del estudiante.

EI FONABE ha resultado un instrumento eficiente, ya que desde su creación en 1997 hasta 2002, su cobertura se incrementó considerablemente. En términos generales, el FONABE asignó en el primer año de funcionamiento un total de 11.768 becas, mientras que en 2002 el fondo otorgó un total de 46.983, lo que representa un incremento del 300\%. Asimismo, en 1997, el monto asignado a los niveles básicos fue de 270 millones de colones, de los cuales 204 millones se asignaron a la educación secundaria y 66 millones a la primaria y educación especial, mientras que las transferencias a la educación superior fueron 
muy reducidas, lo que denota su orientación favorable a estudiantes de los niveles inferiores. La razón principal del reducido presupuesto a la educación superior se debe fundamentalmente a que el FONABE financia solo las titulaciones menos saturadas y consideradas importantes para el desarrollo nacional, para lo cual toma en cuenta las publicaciones del CONARE. En 2002, el presupuesto del FONABE ya ascendía a 3.312 millones de colones (CONARE, 2005). Desafortunadamente no se cuenta con estadísticas actualizadas que permitan determinar el volumen de las ayudas específicas a la educación superior en cuanto al número y cantidad de recursos.

Cada una de las universidades estatales gestiona su propio sistema de becas con los recursos correspondientes al FONABE. Para ello, las universidades determinan dos tipos de becas: a) becas que se otorgan en función de la condición socioeconómica del estudiante, y b) becas de "honor o representación". En relación con las primeras, la UCR y la UNA utilizan para su asignación una escala de 0 a 11. La beca 0 indica que el estudiante debe cubrir el costo total de la matrícula, mientras que con la beca 10 el estudiante queda exento del pago de dicho costo. En cuanto a la beca más alta (11), además de no pagar por la matrícula, el estudiante recibe un monto en especie para facilitar sus estudios. En ambas universidades, la mayoría de los estudiantes becados están en la categoría 9, 10 y 11. EI ITCR y la UNED utilizan una escala de becas diferente (en letras); sin embargo, se pueden realizar comparaciones entre las diferentes escalas. En general, entre un $40 \%$ y un 55\% de los estudiantes matriculados en las universidades públicas se han beneficiado con algún tipo de beca a lo largo del periodo 1990-2003 (Conejo, 2004).

\subsection{Fondos privados}

En Costa Rica, la Ley No 3631 del 7 de diciembre de 1965 establece la política de propiciar oportunidades de educación al mayor número posible de jóvenes que carezcan de recursos y demuestren capacidad para realizar sus estudios. No obstante, las opciones de financiación son realmente escasas para el volumen de estudiantes que se encuentran en alguna de estas situaciones. La escasez de fondos públicos, tal como se expuso en el apartado anterior, no resuelve el problema de financiación de los estudiantes, lo que mina las esperanzas de muchos de ingresar en la enseñanza superior.

Ante tales limitaciones, se crea la Comisión Nacional de Préstamos para la Educación (CONAPE) (Ley No 6041/80), como entidad semi-autónoma destinada a conceder préstamos para la realización de estudios. En la actualidad, la CONAPE brinda financiación a quienes no cuentan con los recursos económicos (preferiblemente de zonas rurales) y su 
prioridad se centra en titulaciones científicas o tecnológicas. Dicha ley establece, en su artículo 20, que los recursos que componen la CONAPE provienen de la recuperación de los préstamos y del $5 \%$ de los beneficios anuales netos de todos los bancos comerciales del país ${ }^{14}$. Además de las funciones meramente financieras, la CONAPE colabora con los beneficiarios en la búsqueda de oportunidades de trabajo acordes con su formación y también ofrece orientación profesional.

Los préstamos de la CONAPE se conceden a los estudiantes para la realización de estudios universitarios, dentro y fuera del país, en cualquiera de las IES, tanto universitarias como no universitarias, públicas y privadas. Entre sus características podemos mencionar: a) las cuotas se realizan una vez finalizados los estudios, b) el tipo de interés es del 15,5\% anual sobre el importe del préstamo, c) cuenta con periodos de carencia, y d) las garantías pueden ser personales, hipotecarias o prendarias, según el monto solicitado.

De acuerdo con la información suministrada por la CONAPE se elabora el Cuadro 6 con los montos asignados durante el periodo 1990-2004.

\section{Cuadro $\mathrm{N}^{\circ} 6$}

Programa de préstamos del CONAPE a la educación superior

\begin{tabular}{|c|c|c|c|}
\hline \hline AÑO & $\begin{array}{c}\text { Número de } \\
\text { beneficiarios }\end{array}$ & $\begin{array}{c}\text { Monto } \\
\text { (USA \$) }\end{array}$ & $\begin{array}{c}\text { Préstamo medio } \\
\text { (USA \$) }\end{array}$ \\
\hline \hline 1990 & 1.254 & 2.844 .425 & 2.268 \\
\hline 1995 & 2.073 & 4.857 .609 & 2.343 \\
\hline 2000 & 2.252 & 7.228 .990 & 3.210 \\
\hline 2003 & 3.561 & 14.993 .252 & 4.210 \\
\hline 2004 & 3.210 & 13.340 .030 & 4.156 \\
\hline \hline Total & $\mathbf{1 2 . 3 5 0}$ & $\mathbf{4 3 . 2 6 4 . 3 0 6}$ & \\
\hline \hline
\end{tabular}

Fuente: Elaboración propia a partir de Conejo (2004)

En el periodo analizado, se beneficiaron con préstamos educativos de la CONAPE un total de 12.350 estudiantes con un monto de 43.264.306 US \$. Si tomamos en cuenta el número de estudiantes matriculados en la educación superior en 2004, obtenemos que menos del $2 \%$ de los estudiantes se benefician de los préstamos CONAPE. Cabe resaltar que de los préstamos otorgados, más del 80\% fue asignado a estudiantes de universidades

\footnotetext{
${ }^{14}$ El monto que los bancos aportan al CONAPE son deducibles del Impuesto sobre la Renta (Ley No 6041/80, artículo 20).
} 
privadas $^{15}$, que además de estos recursos cuentan con otros sistemas propios de financiación de los que disponen algunas de las principales universidades privadas, los cuales operan con características similares a las que ofrece la CONAPE.

En definitiva, no existe en Costa Rica un sistema de ayudas específico para los estudiantes de las universidades públicas, ya que las opciones tienen carácter general para la educación pública (FONABE), y para la educación superior en su conjunto (CONAPE) sin olvidar los préstamos que conceden las universidades privadas a sus estudiantes.

\section{Conclusiones}

El crecimiento experimentado en la matrícula estuvo acompañado de un proceso de diversificación y diferenciación de la oferta de educación superior. En la actualidad, la educación superior costarricense está conformada por instituciones universitarias y no universitarias, que se diferencian a partir de la amplitud de su oferta académica, coexistiendo en ambos subsistemas la oferta de los sectores público y privado. No obstante, la matrícula de educación superior se concentra en más de un 90\% en el sistema universitario, donde las universidades privadas acaparan más de la mitad de dicha demanda. Por tanto, Costa Rica, al igual que el resto de países de la región, ha participado en el proceso de privatización de la educación superior.

Para atender el crecimiento de la demanda, los recursos que reciben las universidades públicas provienen mayoritariamente del FEES, y en menor medida, de recursos procedentes del cobro de matrícula y de la venta de servicios educativos. Por su parte, las universidades privadas no reciben apoyo estatal, lo que supone un mayor esfuerzo en la captación de recursos privados para responder a las necesidades institucionales.

Centrándonos en la financiación de las universidades públicas, la carencia de fondos a mediados de los ochenta, unido a las inequidades en su distribución, introducen las primeras reformas en su modelo de financiación, con el fin de garantizar recursos crecientes a la educación superior. A partir de 1988, se suscriben cada cinco años los Convenios de Financiación, por ello, consideramos que Costa Rica ha sido, en relación a otros países latinoamericanos, pionera en los procesos de planificación e implantación de modelos formales de financiación. En el convenio vigente se define el volumen de los fondos del

\footnotetext{
${ }^{15}$ Se han asignado fundamentalmente a titulaciones de grado (Bachillerato y Licenciatura) en un $97,7 \%$, restando un $2,3 \%$ a las titulaciones de postgrado, mientras que en la educación superior pública, estos se asignan en un 84\% y 16\%, respectivamente (Masís, 2004).
} 
FEES mediante reajuste automático en función del PIB. Así, en el IV Convenio, que rige para el quinquenio 2005-2009, dichas reformas giran en torno a dos líneas de actuación:

\section{- La transformación del FEES}

Contempla dos partidas presupuestarias en función de las actividades que desempeñan las universidades públicas. Así, el FEES está conformado por el FI, donde el gobierno utiliza un modelo incrementalista para asignar los recursos a las actividades básicas de las universidades públicas, los cuales se determinan en función del número de estudiantes y de los costos de los programas, y por el FS, creado en 2005 para asignar recursos a proyectos específicos dirigidos a promover la mejora de la educación superior. En 2007 el FI canaliza a las universidades públicas más del 95\% de los recursos del FEES, mientras que el FS no supera el 5\%.

En nuestra opinión, estos porcentajes reflejan que, a pesar de ser uno de los países pioneros en preocuparse por la implantación de modelos formales de educación, los resultados de la aplicación de tales medidas no distan demasiado de los esquemas de reparto que se encuentran en oros países, esto es, más del $90 \%$ de los fondos públicos que se canalizan a las universidades siguen sistemas puramente incrementalistas. Tales sistemas presentan notables inconvenientes, entre los que destacamos la ausencia de incentivos que vinculen los fondos con las ganancias de eficiencia y calidad en las tareas desempeñadas por las universidades. Por ello, sería recomendable el cambio paulatino de tales esquemas hacia otros que primasen en mayor medida los criterios de eficiencia y eficacia, esto es, modelos basados en fórmulas y contractuales. . ,

En este sentido, el incremento del PIB destinado a la educación superior, así como el porcentaje creciente de los recursos adicionales que se destina al FS, han contribuido a aumentar su participación en el presupuesto del FEES. Este crecimiento puede interpretarse como un esfuerzo financiero por parte de la administración por aumentar el peso de los recursos públicos que se distribuyen a través de criterios de asignación más próximos al mercado y alejarse de modelos puramente incrementalistas. Esta misma tendencia se detecta con la aprobación de fondos orientados a incentivar la mejora de la calidad de la educación superior, como es el caso del Fondo Concursable, el cual adopta un esquema de financiación basado en contratos programa donde las universidades públicas deben competir por dichos recursos. Ambos fondos, FS y Fondo Concursable, de reciente implantación deberían distribuir un volumen mayor de recursos en los próximos años. 


\section{- Diversificación de los recursos}

Los Convenios de Financiación establecen un compromiso por parte de las universidades públicas en la captación de recursos privados a través del cobro de matrícula, de la venta de servicios educativos, de donaciones y otros. Los dos primeros convenios incentivan a las universidades a captar un volumen de recursos privados que, por ejemplo, en 1998 equivalía al 14\% del FEES. Como premio, las instituciones tendrán derecho a una mayor participación en los recursos públicos. Estas condiciones no se establecen en los dos últimos convenios, aunque se mantiene el compromiso en la generación de dichos recursos. Probablemente, ello explica que en 2003 los recursos privados representasen un $15 \%$ del presupuesto de las universidades públicas.

En nuestra opinión, el sector privado ha de contribuir también a la financiación de la educación superior. En este sentido, las universidades han de desempeñar un papel activo en la captación de fondos a través de la comercialización de sus servicios (cursos de reciclaje, asesorías, investigación, desarrollo de patentes, etc.). Un buen sistema para estrechar los vínculos entre universidad y sector productivo es mantener los incentivos para aquellas instituciones que consigan un mayor volumen de recursos privados. Entendemos que dichos incentivos no han de diseñarse como una penalización para las instituciones que no captan suficientes fondos externos sino como un premio para aquellas que son más activas en este frente.

Por su parte, los estudiantes pueden recibir recursos a través de becas y de préstamos educativos. El gobierno emplea fundamentalmente los sistemas de becas para asignar recursos públicos a los estudiantes de la educación superior, aunque estos no constituyen la prioridad del gobierno que se centra más en la financiación de los estudiantes de los niveles básicos.

Por su parte, el instrumento mayormente utilizado para asignar los recursos privados son los préstamos educativos, instrumento fundamentalmente empleado por los matriculados en las universidades privadas (CONAPE), ya que ni estas ni sus estudiantes reciben apoyo estatal. La devolución por parte del estudiante se realiza una vez que este se incorpora al mercado laboral, estableciéndose una cuota que depende de las condiciones del préstamo, como el monto, el plazo y la tasa de interés, por lo que estaríamos en presencia de un préstamo hipotecario. Sin embargo, uno de los inconvenientes que presenta esta modalidad es que desincentiva a los estudiantes que son más reticentes a endeudarse, por lo que resultaría más apropiado el uso de un sistema de préstamo renta donde su devolución 
estaría condicionada a los ingresos futuros percibidos por el graduado una vez incorporado al mercado laboral.

En definitiva, los recursos que se canalizan a los estudiantes vía sistemas de becas y de préstamos suelen ser muy reducidos, siendo necesario promover sistemas más eficaces de ayudas financieras a los estudiantes que contribuyan a incrementar el acceso a la educación superior.

\section{Referencias}

Alvarado, Mayra. (2005). Experiencias de evaluación y acreditación de carreras en Costa Rica. En I Seminario sobre Acreditación y Evaluación de la Educación Superior (pp. 1-10). Guatemala: IESALC.

Araya, Carlos. (2003). Crecimiento, democratización y diversificación de la educación superior en Costa Rica (1970-1994). En Historia de la Educación Costarricense (pp. 365-403). San José: Editorial Universidad Estatal a Distancia-EUNED.

Banco Mundial. (1995): La enseñanza superior: Lecciones derivadas de la experiencia. Washington, D. C., Estados Unidos: El Desarrollo en la Práctica.

Barquero, Jorge, Calvo, Elieth, Ortiz, Marian, Quesada, Iliana, Valverde, Carla y Wachong, Verónica. (2006). Financiamiento de la Educación Superior: Antecedentes y tendencias en el contexto nacional e internacional. San José, Costa Rica: Editorial de la Universidad de Costa Rica.

Brenes, $M^{\mathrm{a}}$. Isabel (2005). Deserción y repitencia de la educación superior universitaria de Costa Rica. Recuperado en junio de 2005, de http://www.iesalc.unesco.org.ve.

Calderón, Ana Lucía (2005). Diagnóstico sobre las políticas de las universidades públicas y privadas de Costa Rica en materia de investigación. En Programa, Diagnóstico de las políticas de investigación universitaria en América Latina y el Caribe (pp. 5157). San José: IESALC/UNESCO.

Cervantes, Flor. (1999). Propuesta de un mecanismo para establecer criterios de distribución del Fondo Especial de la Educación Superior Universitaria Estatal. Revista de Educación, 23 (1), 7-45.

Consejo Nacional de Rectores (CONARE). (2003). Normativa sobre el Financiamiento de la Educación Superior Universitaria Estatal de Costa Rica. Recuperado en octubre de 2005, de http://www.iesalc.unesco.org.ve

Consejo Nacional de Rectores (CONARE). (2005). Estado de la Educación Costarricense. San José, Costa Rica: Programa Estado de la Nación.

Consejo Nacional de Rectores (CONARE). (2006). Plan Nacional de Educación Superior Estatal 2006-2010. Recuperado el 14 de mayo de 2007, de http://www.conare.ac.cr 
Consejo Nacional de Rectores (CONARE). (2007). Fondo Concursable sobre el Estado de la Educación. Recuperado el 21 de mayo de 2007, de http://www.conare.ac.cr.

Conejo, Carlos. (2004). Financiamiento de la Educación Superior en América Latina: El caso de Costa Rica. Recuperado en mayo de 2005, de http://www.iesalc.unesco.org.ve

Costa Rica, Ministerio de Planificación (MIDEPLAN). Sistema de Indicadores sobre Desarrollo Sostenible (SIDES). Recuperado en setiembre de 2005, de http://www.mideplan.go.cr

Guadalupe, Cesar y Louzano, Paula. (2002). Proyecto Regional de Indicadores Educativos de la Cumbre de las Américas. Santiago de Chile, Chile: UNESCO.

Mora, Jorge. (2005). Autoevaluación con fines de acreditación y cultura de la calidad en la educación superior de Costa Rica. En Seminario de la Modernización y Acreditación de la Educación Terciaria [Proyecto BID 1072]. San José: SINAES/IESALC/UNESCO.

Mora, Jorge. (2006). La Educación Superior no Universitaria en Costa Rica. Heredia, Costa Rica: IESALC/UNESCO.

Ruiz, Ángel. (2001a). El destino de Costa Rica y la Educación Superior: El escenario histórico del país, la educación y el papel de la universidad. San José, Costa Rica: Editorial de la Universidad de Costa Rica.

Ruiz, Ángel. (2001b). La Educación Superior en Costa Rica: Tendencias y retos en un nuevo escenario histórico. San José, Costa Rica: Editorial de la Universidad de Costa Rica.

Ruiz, Ángel. (2001c). El siglo XXI y el papel de la universidad: Una radiografía de nuestra época y las tendencias en la Educación Superior. San José, Costa Rica: Editorial de la Universidad de Costa Rica. 